\title{
Análise da camada de fibras nervosas da retina em portadores de enxaqueca com aura
}

\author{
Retinal nerve fiber layer measurements in patients with migraine with aura
}

$\begin{array}{ll}\text { Felipe } & \text { Andrade } \text { Medeiros }^{1} \\ \text { Neuman } & \text { C. Dantas } \\ \text { Maria } & \text { Antonieta Ginguerra } \\ \text { Remo } & \text { Susanna Jr. }\end{array}$

${ }^{1}$ Médico Preceptor do Serviço de Glaucoma do Hospital das Clínicas da Faculdade de Medicina da Universidade de São Paulo (HCFMUSP).

${ }^{2}$ Médica estagiária do Hospital das Clínicas da Faculdade de Medicina da Universidade de São Paulo (HCFMUSP).

${ }^{3}$ Livre-Docente, Chefe do Serviço de Glaucoma do Hospital das Clínicas da Faculdade de Medicina da Universidade de São Paulo (HCFMUSP).

Endereço para correspondência: Av. Brig. Luis Antonio 3030/905 - São Paulo (SP) CEP 01402-000. E-mail: fmedeiros@uol.com.br

\section{RESUMO}

Objetivo: Avaliar as possíveis alterações na camada de fibras nervosas da retina (CFNR) em pacientes com enxaqueca com aura, detectada por meio da polarimetria de varredura a laser. Métodos: Vinte pacientes com enxaqueca com aura e vinte indivíduos normais foram estudados. Os critérios de inclusão para os dois grupos compreenderam: idade de pelo menos 18 anos; ausência de história de doenças oculares, exceto erro refracional ou estrabismo; e ausência de história familiar de hipertensão ocular ou glaucoma. Foram excluídos pacientes com erro refracional maior que 5 DE e/ou 2 DC; acuidade visual corrigida menor que 20/40; pressão intra-ocular maior que $21 \mathrm{mmHg}$; discos ópticos anômalos ou com escavação maior que 0,5 ou assimetria de escavação maior que 0,2 ; ou com presença de doenças retinianas concomitantes. Os pacientes foram submetidos a exame de campo visual (Humphrey 30-2) e análise da CFNR com a polarimetria a laser (GDx - Nerve Fiber Analyzer). Um olho de cada paciente foi randomizado para análise estatística. Resultados: Nenhum dos pacientes do grupo controle apresentou defeitos de campo visual. Nove olhos (45\%) dos pacientes com enxaqueca tiveram anormalidades de campo visual além de $95 \%$ do intervalo de confiança do normal, como indicado pelos índices MD, CPSD ou GHT. Os valores de retardo no setor superior da CFNR dos pacientes com enxaqueca foram significativamente menor que no grupo controle $(\mathrm{p}=0,005)$. Não houve diferença significativa entre os valores de retardo dos dois grupos nas medidas globais e nos setores inferior, nasal e temporal. Conclusão: As medidas de retardo obtidas com a polarimetria a laser foram significativamente menores na porção superior da CFNR dos pacientes com enxaqueca com aura, sugerindo possível dano isquêmico às fibras nervosas, relacionadas à enxaqueca.

Descritores: Fibras nervosas/patologia; Retina; Enxaqueca clássica/complicações; Lasers/ uso diagnostico

INTRODUÇÃO

Desde Hipócrates, em 400 a.C., há menção dos fenômenos visuais que precedem a crise de enxaqueca. Dentre os pródromos visuais freqüentes nestes pacientes, observam-se escotomas cintilantes, fotopsias, hemianopsias uni ou bilaterais, "flashes" luminosos ou linhas coloridas, compondo a chamada aura visual ${ }^{(1)}$. Enquanto os sintomas da aura visual são características bem conhecidas da enxaqueca, um efeito menos conhecido é o desenvolvimento de várias formas de defeitos de campo visual após a crise $^{(2)}$. Embora estes defeitos sejam comumente de natureza transitória ${ }^{(3)}$, durando até sete dias, defeitos de campo visual persistentes ou permanen- 
tes podem ocorrer ${ }^{(4)}$. Alterações permanentes têm sido demonstradas através da perimetria convencional e, em alguns casos, a morfologia dos defeitos sugere acometimento do nervo óptico ${ }^{(5-6)}$. A causa destas alterações provavelmente está relacionada a fenômenos isquêmicos relacionados à enxaqueca.

Sabe-se que defeitos de campo visual são identificados à perimetria convencional somente após um número substancial de fibras nervosas terem sido acometidas ${ }^{(7-8)}$. Desta forma, métodos para avaliação da camada de fibras nervosas da retina (CFNR) têm sido desenvolvidos na tentativa de se identificar déficit estrutural precoce antes do aparecimento de defeitos de campo visual em doenças que lesam o nervo óptico, como o glaucoma ${ }^{(9-10)}$. A polarimetria de varredura a laser permite estimar, de maneira objetiva e quantitativa, a espessura da CFNR in vivo. Seu funcionamento baseia-se na birrefringência da CFNR causada pelo arranjo dos microtúbulos dentro dos axônios das células ganglionares. A birrefringência altera o estado de polarização de um feixe de laser que passa através da CFNR, causando uma medida de retardo que é linearmente correlacionada com a espessura da $\mathrm{CFNR}^{(11)} \mathrm{e}$ corresponde a propriedades bem conhecidas da mesma ${ }^{(12)}$. Esta técnica tem se mostrado promissora na prática clínica podendo diferenciar indivíduos normais de pacientes com glaucoma $^{(13-16)}$, com boa reprodutibilidade entre os exames ${ }^{(17-21)}$.

Sendo assim, o objetivo deste trabalho é estudar, através da polarimetria a laser, as possíveis alterações nas medidas de retardo da camada de fibras nervosas da retina em pacientes com diagnóstico de enxaqueca com aura.

\section{MÉTODOS}

Utilizou-se neste estudo pacientes voluntários oriundos da Liga de Cefaléia do Departamento de Neurologia da Faculdade de Medicina da Universidade de São Paulo, com diagnóstico de enxaqueca com aura segundo a classificação da International Headache Society ${ }^{(22)}$. O grupo controle consistiu de indivíduos saudáveis, pareados por idade, sem história de enxaqueca, recrutados dentre funcionários voluntários da clínica particular de um dos autores (RSJ).

Os critérios de inclusão, para os dois grupos, compreenderam idade de pelo menos 18 anos (sem limite superior), sem antecedentes de qualquer doença ocular, à exceção de erro refracional ou estrabismo, e sem história familiar de hipertensão ocular ou glaucoma. Os pacientes foram submetidos a exame oftalmológico completo que consistiu de refração, exame pupilar, biomicroscopia, medida da pressão intra-ocular com tonômetro de Goldmann e fundoscopia. Foram excluídos do estudo pacientes que apresentassem, em qualquer um dos olhos, erro refracional maior que 5 dioptrias esféricas (DE) ou duas dioptrias cilíndricas (DC); acuidade visual corrigida menor que 20/40; pressão intra-ocular maior que $21 \mathrm{mmHg}$; disco óptico anômalo ou com escavação maior que $0,5 \mathrm{ou}$ assimetria de escavação maior que 0,2 ; ou doenças retinianas. Ametropias menores que as citadas acima parecem não ter influência sobre os resultados do exame ${ }^{(23)}$.
Os pacientes dos dois grupos foram submetidos a exame de campo visual utilizando o programa Full-Threshold 30-2 do perímetro Humphrey Field Analyzer. De cada paciente, foi obtido apenas um exame de campo visual, e o exame de pacientes com enxaqueca foi realizado no mínimo 7 dias após a última crise. Foram excluídos do estudo todos os pacientes que apresentaram campos com baixa confiabilidade, definida como índices de perda de fixação maiores que $20 \%$, ou erros falsopositivos ou falso-negativos maiores que $33 \%$. Do grupo controle, foram ainda excluídos todos os pacientes que apresentaram campo considerado anormal de acordo com os critérios estabelecidos por Anderson.

Os pacientes que satisfizeram todos os critérios acima foram submetidos a exame de análise da camada de fibras nervosas da retina utilizando o aparelho $G D x^{\mathrm{TM}}$ - Nerve Fiber Analyzer (Laser Diagnostic Technologies, Inc., San Diego, CA, Estados Unidos), segundo técnica já descrita ${ }^{(12)}$. Para cada paciente foram obtidas seis imagens de cada olho, sendo que as três melhores foram utilizadas para compor uma imagem média. O desvio-padrão (DP) da imagem média foi de $8 \mu \mathrm{m}$ ou menor para inclusão. De cada imagem média, uma elipse demarcando as bordas do disco óptico foi desenhada, e uma elipse de 10 pixels de espessura, concêntrica à margem do disco óptico e a 1,75 diâmetros discais deste foi automaticamente determinada pelo aparelho. Os parâmetros de retardo global (ao longo de toda a elipse) e quadrânticos (superior, inferior, nasal e temporal) foram então calculados pelo aparelho. Estes parâmetros são fornecidos em micra, representando valores de espessura absoluta da CFNR, após conversão das unidades de retardo para espessura segundo relação obtida previamente em estudo experimental ${ }^{(11)}$. Além destes parâmetros, o software do $G D x^{\mathrm{TM}}$ calcula diversos outros índices de retardo da CFN, incluindo relações entre os setores, cálculos oriundos de rede neural (The Number), dentre outros, cuja sensibilidade e especificidade para diagnóstico do glaucoma foi avaliada por outros autores ${ }^{(24-26)}$. No presente estudo, relatamos apenas os parâmetros de espessura absoluta da CFNR, visto que os outros índices são destinados a análises visando o diagnóstico do glaucoma, sendo em geral relacionados entre si, e fornecendo pouca informação adicional ao propósito deste estudo.

Um olho de cada paciente foi randomizado para análise dos dados de campo visual e análise estatística das medidas de retardo da CFNR. Idade e valores de retardo da CFNR dos pacientes com enxaqueca e do grupo controle foram comparados usando o teste $\mathrm{t}$ de Student. Um valor de $\mathrm{p}$ menor ou igual a 0,05 foi considerado estatisticamente significante.

\section{RESULTADOS}

Foram estudados 20 pacientes com enxaqueca com aura e 20 indivíduos normais. No grupo dos enxaquecosos, 3 indivíduos eram do sexo masculino e 17 eram do sexo feminino, sendo a média de idade \pm dp de $42,8 \pm 17,1$. No grupo controle, 
6 pacientes eram do sexo masculino e 14 do sexo feminino, com média de idade \pm dp de $41,3 \pm 12,9$. O valor de $p$ para a diferença de idade entre os dois grupos foi de 0,76 .

O tempo de história de crises de enxaqueca variou de 1 a 59 anos entre os pacientes, com uma média de 21,1 anos. A freqüência das crises foi bastante variável, cursando de 1 crise ao ano até 5 crises por mês.

A análise dos campos visuais revelou os seguintes resultados: nenhum dos pacientes do grupo controle apresentou defeitos na perimetria computadorizada quando comparados com o banco de dados normativo do aparelho. Nos pacientes com enxaqueca, 9 dos 20 olhos (45\%) apresentaram redução geral de sensibilidade (MD) além de $95 \%$ do intervalo de confiança do normal; cinco olhos (20\%) apresentaram CPSD (medida da irregularidade do campo visual sugestiva de escotoma) além de $95 \%$ do intervalo de confiança do normal; e 6 olhos tiveram GHT classificado como fora dos limites da normalidade ou "borderline".

A tabela 1 lista as médias $\pm \mathrm{dp}$ dos valores de retardo da camada de fibras nervosas, bem como os valores de probabilidade para as diferenças entre os dois grupos. Houve uma diferença estatisticamente significante entre os pacientes com enxaqueca e o grupo controle no quadrante superior $(\mathrm{p}=0,005)$, com menores valores obtidos nos pacientes com enxaqueca. As medidas de retardo global e nos quadrantes inferior, nasal e temporal foram menores no grupo de pacientes com enxaqueca, embora a diferença não tenha atingido significância estatística. A figura 1 mostra a distribuição dos valores de retardo encontrados nos dois grupos, em um gráfico do tipo "box plot".

\section{DISCUSSÃO}

Os déficits visuais permanentes podem ocorrer em qualquer paciente que experimenta a aura visual da enxaqueca. Lewis et al. ${ }^{(5)}$ identificaram alguma forma de perda de campo visual em 21 de 60 (35\%) pacientes com enxaqueca, sendo a mais comum à depressão generalizada de campo visual. No nosso trabalho, encontramos proporção ainda maior de alteração, sendo que o achado mais comum também foi o de diminuição generalizada de sensibilidade. Em alguma extensão, esta alta taxa poderia ser atribuída a seleção de

\begin{tabular}{|lccc|}
\hline $\begin{array}{c}\text { Tabela 1. Valores de espessura da camada de fibras nervosas } \\
\text { da retina: média } \pm \text { dp } \\
\text { (em micra) nos pacientes com enxaqueca } \\
\text { e no grupo controle }\end{array}$ \\
$\begin{array}{l}\text { Enxaqueca } \\
(\mathbf{n = 2 0 )}\end{array}$ & $\begin{array}{c}\text { Normais } \\
(\mathbf{n = 2 0 )}\end{array}$ & $\begin{array}{c}\text { P } \\
\text { (teste } \mathbf{t} \text { de Student) }\end{array}$ \\
Global & $60,90 \pm 6,223$ & $64,35 \pm 8,493$ & 0,152 \\
Superior & $83,60 \pm 14,16$ & $98,70 \pm 18,25$ & 0,005 \\
Inferior & $86,90 \pm 14,16$ & $91,15 \pm 13,28$ & 0,272 \\
Nasal & $46,84 \pm 6,399$ & $51,41 \pm 8,190$ & 0,057 \\
Temporal & $39,24 \pm 9,734$ & $40,68 \pm 9,408$ & 0,636 \\
\hline
\end{tabular}

pacientes referidos por neurologistas, pois eles poderiam representar uma forma mais severa de enxaqueca. Além disso, cada paciente realizou apenas um exame de campo visual, sendo então difícil determinar a reprodutibilidade dos achados.

Os déficits visuais permanentes podem resultar de dano a qualquer parte do sistema visual, incluindo córtex, quiasma óptico, retina e coróide ${ }^{(27)}$. Classicamente, tem-se relacionado o tipo de defeito com a região acometida: defeitos homônimos por isquemia do córtex estriado, defeitos hemianópicos bitemporais por isquemia do quiasma, e defeitos de morfologia não localizatória sugestiva de acometimento da retina ou nervo óptico. Vários estudos relatando envolvimento de campo visual em pacientes com enxaqueca encontraram uma alta proporção de indivíduos com defeitos de campo visual unilaterais, sugestivos de dano pré-cortical ${ }^{(5,28-30)}$. Lewis et al. ${ }^{(5)}$ encontraram defeitos unilaterais em 14 dos 21 pacientes afetados. De maneira similar, De Natale et al. ${ }^{(30)}$ relataram envolvimento unilateral em 10 de 17 casos com perda de campo visual. Contudo, a causa do envolvimento pré-cortical na enxaqueca não está clara. Existem vários relatos na literatura descrevendo dano ao nervo óptico como resultado de eventos isquêmicos relacionados à enxaqueca, se manifestando como neuropatia óptica isquêmica anterior ou posterior ${ }^{(31-33)}$. No entanto, estes eventos têm sido considerados incomuns. Presumivelmente, a redução do fluxo sanguíneo para o nervo óptico durante uma crise enxaquecosa raramente cai a um nível suficientemente baixo a ponto de se manifestar como um infarto franco, resultando no quadro típico de neuropatia óptica isquêmica. Contudo, poderíamos especular que ataques de enxaqueca com isquemia importante poderiam dar origem a alguma forma de dano ao nervo óptico não detectada através de exames convencionais, mas potencialmente demonstrável através da análise da camada de fibras nervosas da retina.

Vários autores têm sugerido que anormalidades vasculares podem estar presentes na vasculatura periférica de pacientes com história de crises de enxaqueca ${ }^{(34-36)}$. Suporte a estes achados é fornecido pela observação de que pacientes com enxaqueca apresentam uma maior incidência de condições de origem presumivelmente vascular como glaucoma de pressão normal $^{(37-39)}$, fenômeno de Raynaud ${ }^{(40,41)} \mathrm{e}$ angina variante ${ }^{(42,43)}$. A enxaqueca, juntamente com estas outras condições, comporia a chamada síndrome vasoespástica ${ }^{(44)}$.

Flammer ${ }^{(44)}$ descreveu pacientes com síndrome vasoespástica e defeitos de campo visual semelhantes aos de pacientes com glaucoma, mas sem alterações no nervo óptico ou em outros exames oftalmológicos ou neurológicos, sugerindo que os vasoespasmos oculares seriam os responsáveis pelo déficit funcional. McKendrick et al. ${ }^{(28)}$ especularam que em pacientes com ataques repetidos de enxaqueca, os vasoespasmos periféricos ocorrendo na região do nervo óptico ou coróide circundante, poderiam eventualmente levar a dano estrutural.

Neste estudo, os valores de retardo do quadrante superior da camada de fibras nervosas da retina foram significativa- 
mente menores nos indivíduos com enxaqueca que nos sujeitos normais. É bem estabelecido que as neuropatias ópticas isquêmicas não arteríticas (NOINA) comumente se manifestam com defeitos inferiores de campo visual, que correspondem à perda superior de fibras nervosas ${ }^{(45-48)}$. Quigley et al. ${ }^{(49)}$, estudando as fibras remanescentes postmortem do nervo óptico de 3 indivíduos com história prévia de NOINA, mostraram uma perda de fibras completa na metade superior de cada nervo óptico, incluindo também uma perda de fibras periféricas na outra metade. Assim, a isquemia do nervo óptico relacionada à enxaqueca seguiria um padrão similar de dano, embora se manifestando menos comumente com defeitos de campo visual.

A análise do gráfico de distribuição dos valores de retardo da CFNR (Figura 1) mostra que há uma grande sobreposição dos valores entre os pacientes normais e os enxaquecosos, mesmo no setor superior. A grande variabilidade das características da enxaqueca em cada paciente, e também as variações individuais na susceptibilidade do nervo óptico a eventos isquêmicos, poderiam se relacionar aos diferentes graus de perda de fibras nervosas detectadas nestes pacientes.

Por fim, a patogenia do dano neuronal isquêmico na enxaqueca é ainda um evento complexo e pouco elucidado, sendo necessários maiores estudos para fornecer evidência inequívoca da relação entre a isquemia relacionada à enxaqueca, e a perda de fibras nervosas detectada pelo $G D x^{\mathrm{TM}}$ nesses pacientes.

\section{ABS TRACT}

Purpose: To evaluate the possible alterations of the retinal nerve fiber layer (RNFL), as detected by scanning laser po-

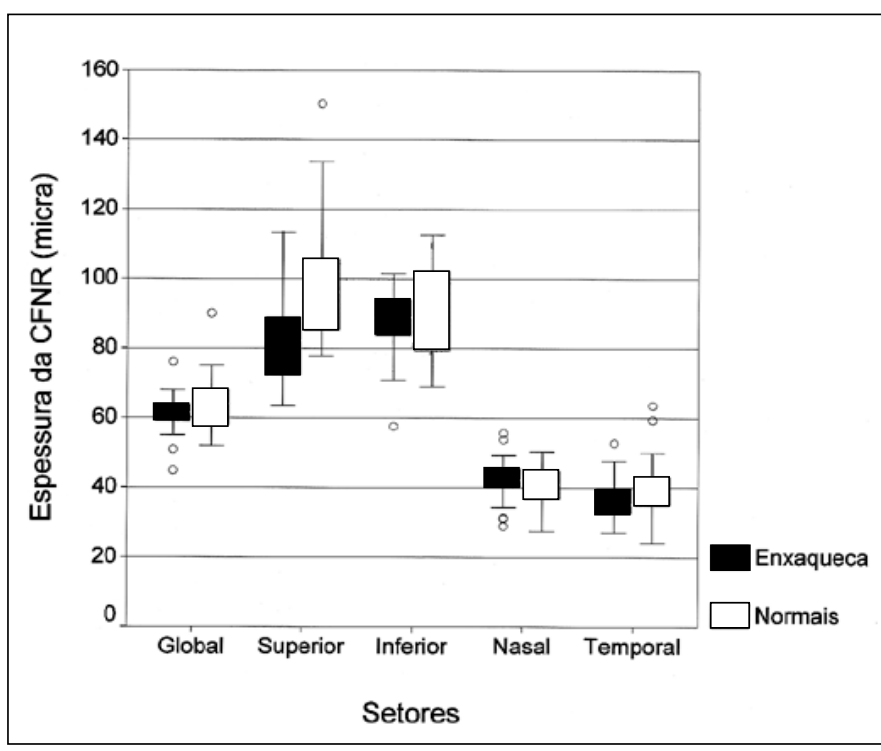

Figura 1 - Gráfico tipo "box plot" mostrado a distribuição dos valores de espessura da camada de fibras nervosas da retina nos pacientes com enxaqueca e no grupo controle larimetry (SLP), that may occur in patients with migraine with aura. Methods: Twenty patients with migraine with aura and twenty normal subjects were studied. Inclusion criteria for the two groups included age of at least 18 years, no history of any ocular disease except refractive error or strabismus, and no family history of ocular hypertension or glaucoma. Potential subjects underwent a comprehensive eye examination. Exclusion criteria included refractive error greater than $5 \mathrm{DS}$ and/or 2 DC, best-corrected visual acuity less than 20/40, intraocular pressure greater than $21 \mathrm{mmHg}$, abnormal optic discs, cup-todisc ratio $(\mathrm{C} / \mathrm{D})$ greater than 0.5 or asymmetry between $\mathrm{C} / \mathrm{D}$ of the two eyes greater than 0.2 , or any other concomitant retinal pathology. The patients were submitted to a Humphrey Field (30-2) examination and RNFL analysis using the GDx Nerve Fiber Analyzer. One eye from each patient was randomly selected for statistical analysis. Results: None of our controls had any visual field defects. Nine eyes (45\%) from migraine patients had visual-field abnormalities beyond $95 \%$ normal confidence limits, as indicated by MD, CPSD or GHT. The retardation values of the superior nerve fiber layer of migraine patients were significantly lower than values in normal patients $(\mathrm{p}=0.005)$. No significant differences were observed in global, inferior, temporal and nasal measurements between the two groups. Conclusions: In this study, the RNFL measurements were significantly lower in the superior retina of migraine patients, indicating that these patients might have migraine-related ischemic damage of nerve fibers.

Keywords: Nerve fibers/pathology; Retina; Migraine, classic / complications; Lasers/diagnostic use

\section{REFERENCIAS}

1. Queiroz LP, Rapoport AM, Weeks RE, Sheftell FD, Siegel SE, Baskin SM. Characteristics of migraine visual aura. Headache 1997;37:137-41.

2. Galezowski X. Ophtalmic migrain. Lancet 1882;1:176.

3. Ebner R. Visual field examination during transient migrainous visual loss. J Clin Neuro-ophthalmol 1991;11:114-7.

4. Hupp SL, Kline LB, Corbett JJ. Visual disturbances in migraine. Surv Ophthalmol 1989;33:221-36.

5. Lewis RA, Vijayan N, Watson C, Keltner J, Johnson CA. Visual loss in migraine. Ophthalmology 1989;96:321-6.

6. Susanna Junior R. Enxaqueca oftálmica - alterações do campo visual na crise. Rev Bras Oftalmol 1990;49:297-302.

7. Quigley HA, Dunkelberger GR, Green WR. Retinal ganglion cell atrophy correlated with automated perimetry in human eyes with glaucoma. Am J Ophthalmol 1989;107:453-64.

8. Kerrigan-Baumrind LA, Quigley HA, Pease ME, Kerrigan DF, Mitchell RS. Number of ganglion cells in glaucoma eyes compared with threshold visual field tests in the same persons. Invest Ophthalmol Vis Sci 2000;41:741-8.

9. Sommer A, Miller NR, Pollack I, Maumenee AE, George T. The nerve fiber layer in the diagnosis of glaucoma. Arch Ophthalmol 1977;95:2149-56.

10. Sommer A, Katz J, Quigley HA, Miller NR, Robin AL, Richter RC et al. Clinically detectable nerve fiber atrophy precedes the onset of glaucomatous field loss. Arch Ophthalmol 1991;109:77-83.

11. Weinreb RN, Dreher AW, Coleman A, Quigley H, Shaw B, Reiter K. Histopathologic validation of Fourier-ellipsometry measurements of retinal nerve fiber layer thickness. Arch Ophthalmol 1990;108:557-60.

12. Weinreb RN, Shakiba S, Zangwill L. Scanning laser polarimetry to measure the nerve fiber layer of normal and glaucomatous eyes. Am J Ophthalmol 1995;119:627-36. 
13. Weinreb RN, Shakiba S, Sample P, Shahrokni S, van Horn S. Garden VS et al. Association between quantitative nerve fiber layer measurement and visual field loss in glaucoma. Am J Ophthalmol 1995;120:732-8.

14. Susanna Junior R, Takahashi WY, Nakamura NF. Sensibilidade e especificidade da avaliação da camada de fibras nervosas examinadas através da polarimetria a laser. Rev Bras Oftalmol 1998;57:17-22.

15. Tjon-Fo-Sang MJ, de Vries J, Lemij HG. Measurement by nerve fiber analyzer of retinal nerve fiber layer thickness in normal subjects and patients with ocular hypertension. Am J Ophthalmol 1996;122:220-7.

16. Anton A, Zangwill L, Emdadi A, Weinreb RN. Nerve fiber layer measurements with scanning laser polarimetry in ocular hypertension. Arch Ophthalmol 1997;115:331-4.

17. Zangwill L, Berry CA, Garden VS, Weinreb RN. Reproducibility of retardation measurements with the nerve fiber analyzer II. J Glaucoma 1997;6:384-9.

18. Hoh ST, Ishikawa H, Greenfield DS, Liebmann JM, Chew SJ, Ritch R. Peripapillary nerve fiber layer thickness measurement reproducibility using scanning laser polarimetry. J Glaucoma 1998;7:12-5.

19. Duarte FB, Paranhos Junior A, Galhardo RA, Abdanur LRA, Prata Junior JÁ, Mello PAA. Reprodutibilidade do exame de medida da camada de fibras nervosas da retina por meio da polarimetria de varredura a laser. Arq Bras Oftalmol 1999;62:555-60.

20. Medeiros FA, Leal BC, Susanna Junior R. Efeito do tamanho pupilar na medida de espessura da camada de fibras nervosas da retina com a polarimetria a laser. Rev Bras Oftalmol 2000;59:639-46.

21. Colen TP, Tjon-Fo-Sang MJ, Mulder PG, Lemij HG. Reproducibility of measurements with the nerve fiber analyzer (NFA/GDx). J Glaucoma 2000;9: 363-70.

22. Classification and diagnostic criteria for headache disorders, cranial neuralgias and facial pain. Headache Classification Commitee of the International Headache Society: Cephalalgia 1988;8(Suppl 7):1-96.

23. Alvarenga LS, Moura CR, Mello PAA, Prata Junior JA. Equivalente esférico e valores da espessura da camada de fibras nervosas obtidas com o $G D x^{\mathrm{TM}}$ Scanning Laser System ${ }^{\circledR}$. Arq Bras Oftalmol 1999;62:693-6.

24. Trible JR, Schultz RO, Robinson JC, Rothe TL. Accuracy of scanning laser polarimetry in the diagnosis of glaucoma. Arch Ophthalmol 1999;117:1298-304.

25. Weinreb RN, Zangwill L, Berry CC, Bathija R, Sample PA. Detection of glaucoma with scanning laser polarimetry. Arch Ophthalmol 1998;116:1583-9.

26. Paczka JA, Quigley HA, Friedman DS. Capabilities of frequency-doubling technology, scanning laser polarimetry and nerve fiber layer photographs to distinguish glaucomatous damage [ARVO Abstract]. Invest Ophthalmol Vis Sci 1999;40:S841.

27. Kupersmith MJ, Warren FA, Hass WK. The non-benign aspects of migraine. Neuro-ophthalmology 1987;7:1-10.

28. McKendrick AM, Vingrys AJ, Badcock DR, Heywood JT. Visual field losses in subjects with migraine headaches. Invest Ophthalmol Vis Sci 2000;41: 1239-47.

29. Nizankowska MH, Turno-Krecicka A, Misiuk-Hojlo M, Ejma M, Chelstowsjka J, Szczesna-Borzemska D et al. Coexistence of migraine and glaucoma-like visual field defects. Klin Oczna 1997;99:121-6.
30. De Natale R, Polimeni D, Narbone MC, Scullica MG, Pelicano M. Visual field defects in migraine patients. In: Mills RP, editor. Perimetry update 93/ 94. Amsterdam: Kugler; 1994. p. 283-4.

31. Lana MA, Mattos Junior RM. Neuropatia óptica isquêmica associada à enxaqueca. Arq Bras Oftalmol 1998;61:276-84.

32. Katz B, Bamford CR. Migrainous ischemic optic neuropathy. Neurology $1985 ; 35: 112-4$

33. Weinstein JM, Feman SS. Ischemic optic neuropathy in migraine. Arch Ophthalmol 1982;100:1097-100.

34. Gasser P, Flammer J, Guthauser U, Mahler F. Do vasospasms provoke ocular disease? Angiology 1990;41:213-20.

35. Drance SM, Douglas GR, Wijsman K, Schultzer M, Britton RJ. Response of blood flow to warm and cold in normal and low-tension glaucoma patients. Am J Ophthalmol 1988;105:35-9.

36. Hegyalijai T, Meienberg O, Dubler B, Gasser P. Cold-induced acral vasospasm in migraine as assessed by nailfold video-microscoscopy: prevalence and response to migraine prophylaxis. Angiology 1997;48:345-9.

37. Phelps CD, Corbett JJ. Migraine and low-tension glaucoma. A case-control study. Invest Ophthalmol Vis Sci 1985;26:1105-8.

38. Corbett JJ, Phelps CD, Eslinger P, Montague PR. The neurologic evaluation of patients with low-tension glaucoma. Invest Ophthalmol Vis Sci 1985;26: 1101-4.

39. Cursiefen C, Wisse M, Cursiefen S, Junemann A, Martus P, Korth M. Migraine and tension headache in high-pressure and normal-pressure glaucoma. Am J Ophthalmol 2000;129:102-4.

40. O'Keffe ST, Tsapatsaris NP, Beetham WP. Increased prevalence of migraine and chest pain in patients with primary Raynaud disease. Ann Intern Med 1992;116(12 pt 1):985-9

41. Zahavi I, Chagnac A, Hering R, Davidovich S, Kuritzky A. Prevalence of Raynaud's phenomenon in patients with migraine. Arch Intern Med 1984; 144:742-4.

42. Wayne VS. A possible relationship between migraine and coronary artery spasm. Aust N Z J Med 1986;16:708-10.

43. Lafitte C, Even C, Henry-Lebras F, de Toffol B, Autret A. Migraine and angina pectoris by coronary artery spasm. Headache 1996;36:332-4.

44. Flammer J. To what extent are vascular factors involved in the pathogenesis of glaucoma? In: Kaiser HJ, Flammer J, Hendrickson Ph, editors. Ocular blood flow. Basel: Karger; 1996. p. 12-39.

45. Boghen DR, Glaser JS. Ischemic optic neuropathy: the clinical profile and natural history. Brain 1975;98:689-708.

46. Hayreh SS, Podhajsky P. Visual field defects in anterior ischemic optic neuropathy. Doc Ophthalmol Proc Ser 1979;19:53-71.

47. Repka MX, Savino PJ, Schatz NJ, Sergott RC. Clinical profile and long-term implications of anterior ischemic optic neuropathy. Am J Ophthalmol 1983; 96:478-83.

48. Rizzo JF, Lessell S. Optic neuritis and ischemic optic neuropathy. Overlapping clinical profiles. Arch Ophthalmol 1991;109:1668-72.

49. Quigley HA, Miller NR, Green WR. The pattern of optic nerve fiber loss in anterior ischemic optic neuropathy. Am J Ophthalmol 1985;100:769-76.

\section{Novidades na Internet!!!}

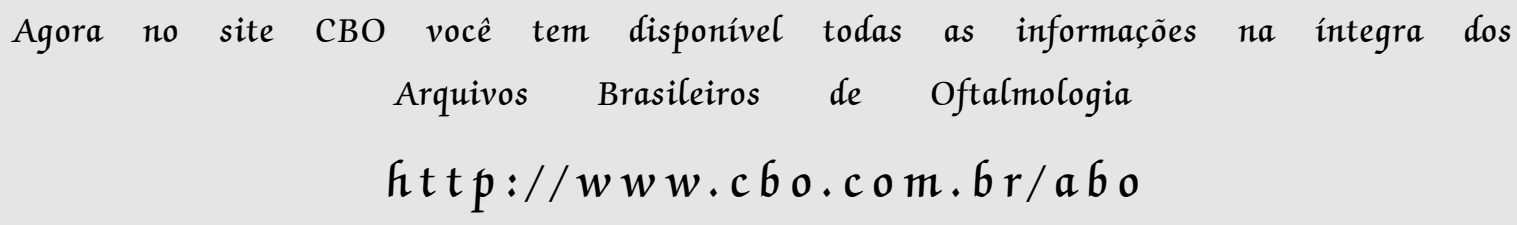

